\section{A PHOTO-ELECTRIC FOURIER TRANSFORMER}

By Prof. MAX BORN, F.R.S., DR. R. FÜRTH, and R. W. PRINGLE

Department of Mathematical Physics, University of Edinburgh

HE 'Fourier transform' $g(y)$ of a function $f(x)$, usually defined by the integral

$$
g(y)=\frac{1}{\sqrt{2 \pi}} \int_{a}^{b} f(x) e^{-i y x} d x, . \quad . \quad .
$$

plays an important part in many problems of pure and applied physics. It represents, for example, the connexion between the intensity distribution of a wave scattered by matter of a certain density distribution, which has to be calculated in a number of acoustical and optical problems and, above all, in $\mathrm{X}$-ray crystal analysis work. It further allows the resolution of a complicated oscillation into a continuous frequency spectrum of harmonic oscillations, which is required in many problems of mechanical and electrical engineering. It therefore seems of some importance to have an instrument by which the Fourier transform of a given function can be automatically and quickly produced. We have now succeeded in building up an instrument which produces the graph of the function

$$
g^{\prime}(y)=\int_{a}^{b} f(x) \cos (y x+\delta) d x . . .
$$

on the screen of a cathode ray oscillograph, from a mask cut out of black paper in the shape of the graph of the function $f(x)$, or from a record of this function on a plate or film in density variation. Obviously two of the functions $g^{\prime}(y)$ for two values of $\delta$, say, $\delta=0$ and $\delta=\pi / 2$, are equivalent to the complex function $g(y)$ when $f(x)=0$ for $x<a, x>b$.

The method is a photo-electric one, based on a similar principle to that used by Montgomery for discrete Fourier analysis, but extended for continuous Fourier analysis, that is, Fourier integration. A pattern of parallel fringes, with the light intensity varying according to a harmonic function in a direction normal to the fringes, is projected on the mask (or record) mentioned above representing the given function $f(x)$, and the transmitted light is concentrated on the cathode of an electron-multiplier vacuum photocell. The anode current through the cell will then obviously be proportional to the righthand side of the expression (2) for a given spacing $2 \pi / y$ and setting $\delta$ of the fringe system. $y$ is now made to vary periodically in time, that is, the fringe system is made to extend and shrink periodically in the following way.

A glass disk, carrying a photographically produced fringe pattern in which the transparency varies sinoidally, is rotated about an axis perpendicular to its own plane. It can be set so as to let the axis intersect the pattern either at a point of minimum density $(\delta=0)$, or maximum density $(\delta=\pi)$, or half-way between two such points $(\delta=\pi / 2)$, or for any other phase required. The disk is evenly illuminated with light from a projector lamp, and a real image of the fringes is produced on an opaque screen with a narrow adjustable slit in it, the axis of rotation of the image passing precisely through the centre of the slit. The light intensity along the slit will
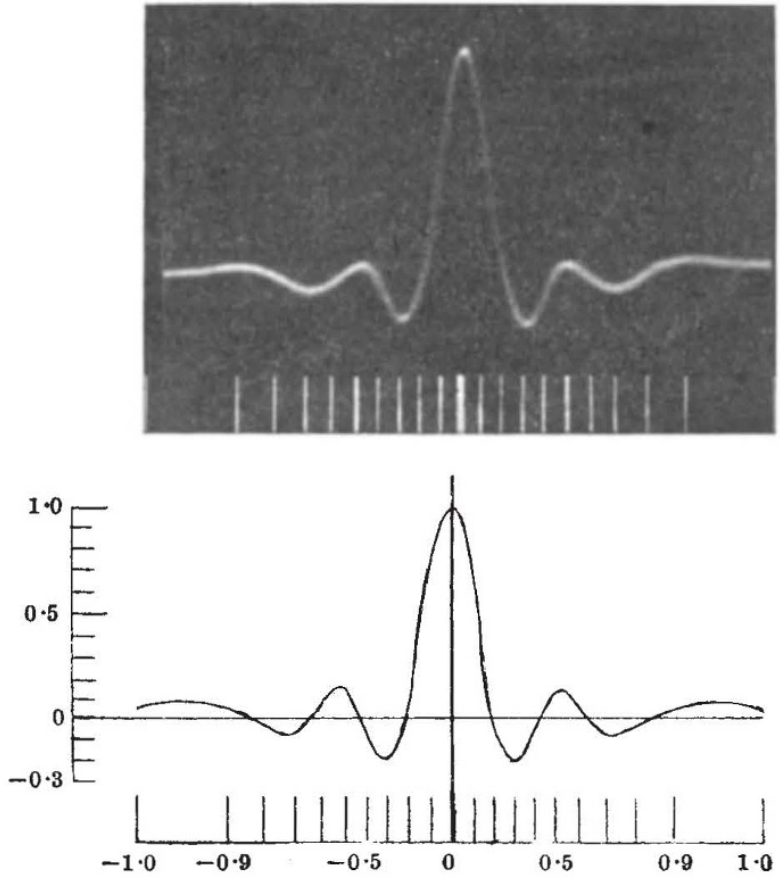

Fig. 1. Above : Record of the Fourier transform

$$
g^{\prime}(y)=\int_{0}^{a} c \cos y x d x \approx \frac{\sin a y}{y}
$$

Below : Calculated graph of the same function.

then be a sinoidal one with a spacing which shrinks and extends periodically in time as the disk rotates. Finally, by projecting an image of the slit on the plane of the mask (or record) by means of a cylindrical lens, with its cylinder axis at right angles to the slit, the optical arrangement is completed.

In order to produce the graph of the Fourier transform, the voltage across a resistance in the anode circuit of the photocell is transferred to a cathode ray oscillograph with which is incorporated a twostage linear amplifier, and a harmonic time base is supplied, synchronized to the rotation of the disk. As the replacement of one mask (or record) by another one, the setting of the phase, and the photographing of the trace take only a couple of minutes, the new method is apparently capable of producing the required Fourier transforms in a minimum of time.

Figs. 1 and 2 show some examples of the performance of the instrument. The records were produced by using the incorporated linear time base of the oscillograph, instead of a harmonic time base, which has the effect of making the horizontal scale of the graphs non-linear. The correct scale has been inserted at the bottom of each graph. Fig. I shows (above) the actual record of the Fourier transform $g^{\prime}(y)=\int^{a} C \cos y x d x \approx \frac{\sin a y}{y}$ and (below) the calculated graph of this function, and gives an idea of the accuracy of the instrument. Fig. 2 shows the records of four other Fourier transforms. We have satisfied ourselves also that in these and many other cases the curves obtained coincide with the calculated ones within the limits of accuracy of the present instrument. 


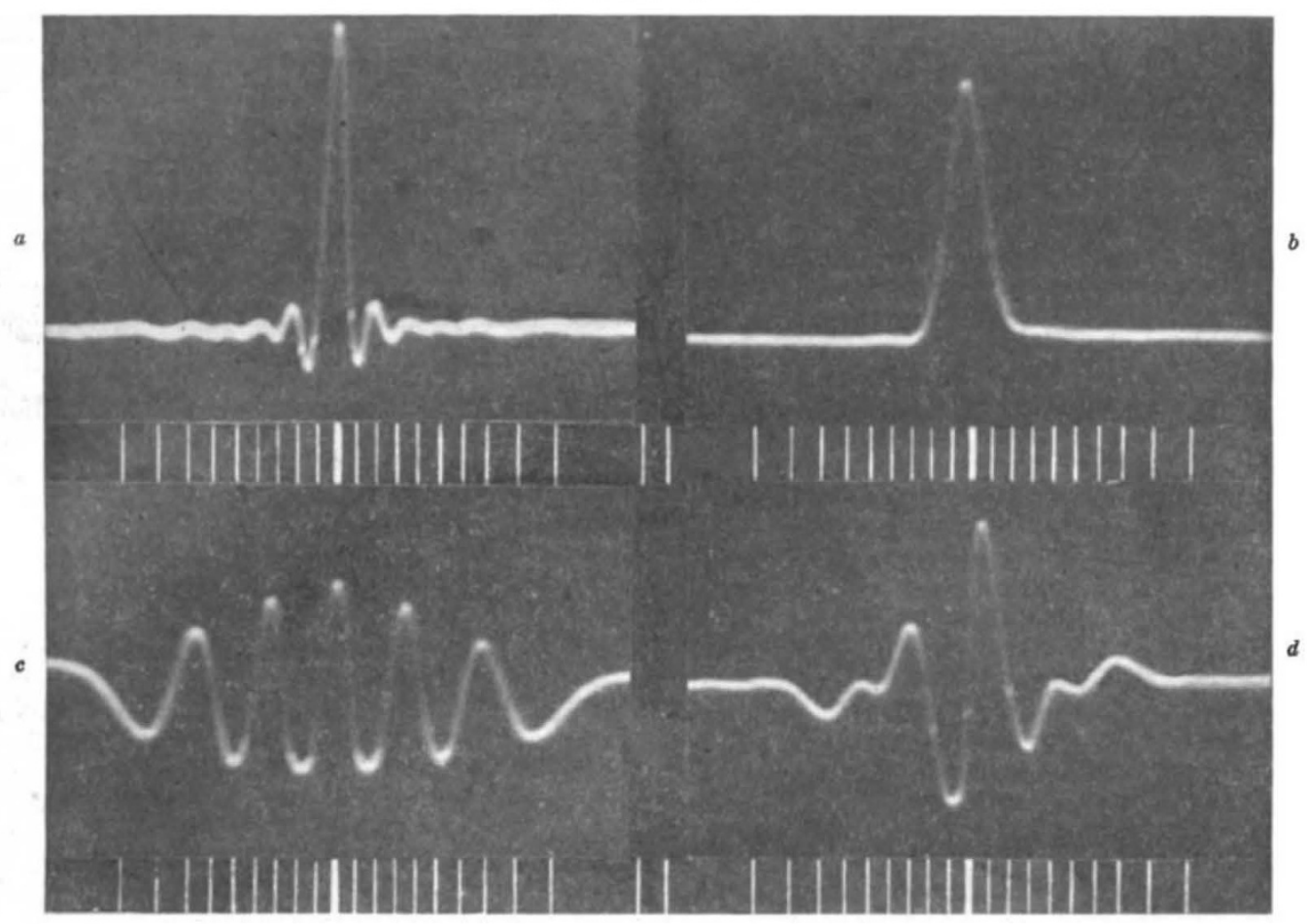

Fig. 2. Records of the Fourier transforms
(a) $g^{\prime}(y)=\int_{-A}^{+A} C \cos y x d x \approx \frac{\sin A y}{y} ;(A=2 \cdot 3 a) ;$
(c) $g^{\prime}(y)=\int_{a}^{a+\varepsilon} C \cos y x d x \sim \frac{\cos a y \cdot \sin 8 y / 2}{y}(\varepsilon=a / b) ;$

There is little doubt that further technical develop. ment of the instrument will make possible the production of Fourier transforms with any reasonably desired accuracy. A detailed description of the new method will shortly be given elsewhere.

\section{THE NEWCOMEN SOCIETY}

$\mathrm{T}$

HE jubilee dinner of the Newcomen Society held at Kettner's Restaurant, Romilly Street, W.I, on November 14, when a presentation was made to Dr. H. W. Dickinson, the chief guest of the evening, for his twenty-five years work for the Society, will long be remembered by those present. It was on November 5, 1920, in the Court Room of H.M. Patent Office, that the Society held its first general meeting, and Dr. Dickinson has ever since been an honorary secretary, except for the two years of his presidency. During this period he has contributed many papers to the Proceedings, arranged all the summer meetings and edited the whole of the Transactions, while the contacts he made during his visits to the United States in 1923 and 1938 had much to do with the rapid growth of the Society there, a growth which has led to the formation of a North American Branch as an autonomous affiliated society with a membership exceeding five thousand.

The Society came into existence through a chance conversation between Mr. A. Titley and the late Mr. J. W. Hall on September 16, 1919, at the garden (b) $\begin{aligned} g^{\prime}(y) & +\int_{-A}^{+A} e^{-b x^{2} / 2} \cos y x d x \sim e^{-y^{2} / 2 b} ;\end{aligned}$

$3 a / 2$

(d) $g^{\prime}(y)=\int_{a / 2}^{3 a / 2} C \sin y x d x \approx \frac{\cos a y / 2-\cos 3 a y / 2}{y}$

party in the grounds of Heathfield Hall, held during the Birmingham commemoration of the centenary of the death of James Watt. This gathoring had attracted many engineers with a love of history, and the suggestion of Mr. Titley to the effect that a society should be formed for furthering the study of the history of engineering and technology at once gained support. By May 1920 a provisional committee had been formed at Birmingham, but the Society may be said to have made its debut at a meeting a month later, on June 4, in the offices of Mr. L. St. L. Pendred, editor of the Engineer, at 33 Norfolk Street, London, and at a dinner held the same evening at the Savage Club, then in the Adelphi. At those gatherings Mr. Titley was chosen as president, Dr. (then Mr.) Dickinson, honorary secretary, while Messrs. Pendred, Rhys Jenkins and A. W. Hulme were to act as the London committee to collaborate with the Birmingham committee. After much discussion the question of a name for the Society was happily solved by the adoption of the surname of 'the father of the steam engine', Thomas Newcomen, of Dartmouth. Though its membership was small, the Society soon got into its stride, and at the meeting on November 5, 1920, Mr. Hulme read his paper entitled "Introduction to the Literature of Historical Engineering to the Year 1640", which forms a good starting point for any student eager to take up technological history. It appears as the first paper in vol. 1 of the Transactions, which contains also two of Mr. Rhys Jenkins' many papors to the Society, Mr. Pendred's "The Mystery of 